\title{
XLEnt: Mining a Large Cross-lingual Entity Dataset with Lexical-Semantic-Phonetic Word Alignment
}

\author{
Ahmed El-Kishky ${ }^{1}$ Adithya Renduchintala ${ }^{2}$ James Cross $^{2}$ \\ Francisco Guzmán ${ }^{2}$ Philipp Koehn ${ }^{3}$ \\ ${ }^{1}$ Twitter Cortex, ${ }^{2}$ Facebook AI ${ }^{3}$ Johns Hopkins University \\ aelkishky@twitter.com, \{adirendu,jcross,fguzman\}@fb.com, phi@jhu.edu
}

\begin{abstract}
Cross-lingual named-entity lexica are an important resource to multilingual NLP tasks such as machine translation and cross-lingual wikification. While knowledge bases contain a large number of entities in high-resource languages such as English and French, corresponding entities for lower-resource languages are often missing. To address this, we propose LexicalSemantic-Phonetic Align (LSP-Align), a technique to automatically mine cross-lingual entity lexica from mined web data. We demonstrate LSP-Align outperforms baselines at extracting cross-lingual entity pairs and mine 164 million entity pairs from 120 different languages aligned with English. We release these cross-lingual entity pairs along with the massively multilingual tagged named entity corpus as a resource to the NLP community.
\end{abstract}

\section{Introduction}

Named entities are references in natural text to real-world objects such as persons, locations, or organizations that can be denoted with a proper name. Recognizing and handling these named entities in many languages is a difficult, yet crucial, step to language-agnostic text understanding and multilingual natural language processing (NLP) (Sekine and Ranchhod, 2009).

As such, cross-lingual named entity lexica can be invaluable resources towards making tasks such as entity linking, named entity recognition (Ren et al., 2016b,a), and information and knowledge base construction (Tao et al., 2014) inherently multilingual. However, the coverage of many such multilingual entity lexica (e.g., Wikipedia titles) is less complete for lower-resource languages and approaches to automatically generate them under-perform due to the poor performance of low-resource taggers (Feng et al., 2018; Cotterell and Duh, 2017).

To perform low-resource NER, previous efforts have applied word alignment techniques to project

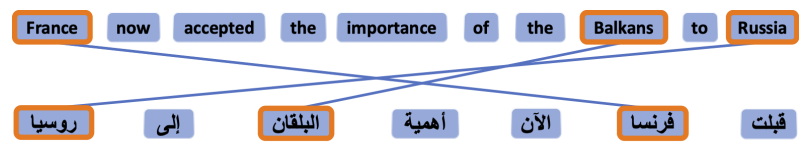

Figure 1: Identify entity pairs by projecting English entities onto lower-resource languages via word-alignment.

available labels to other languages. Kim et al. (2010) applies heuristic approaches with alignment correction using an alignment dictionary of entity mentions. Das and Petrov (2011) introduced a novel label propagation technique that creates a tag lexicon for the target language, while Wang and Manning (2014) instead projected model expectation rather than labels thus transferring word boundary uncertainty. Additional work jointly performs word alignment while training bilingual name tagging (Wang et al., 2013); however this method assumes the availability of named entity taggers in both languages. Other methods have leveraged bilingual embeddings for projection (Ni et al., 2017; Xie et al., 2018).

In this work, we propose using named-entity projection to automatically curate a large crosslingual entity lexicon for many language pairs. As shown Figure 1, we construct this resource by performing NER in a higher-resource language, then projecting the entities onto text in a lowerresource language using word-alignment models.

Our main contribution is the construction and release of a large web-mined cross-lingual entity dataset that will be beneficial to the NLP community. Our proposed alignment model, LSP-Align, principally combines the lexical, semantic, and phonetic signals to extract higher-quality cross-lingual entity pairs as verified on a ground-truth entity pair set. With LSP-Align, we mined over $164 M$ distinct cross-lingual entity pairs spanning 120 language pairs and freely release the XLEnt dataset ${ }^{12}$ in hope it spurs further work in cross-lingual NLP.

\footnotetext{
${ }^{1}$ http://data.statmt.org/xlent/

${ }^{2}$ https://opus.nlpl.eu/XLEnt-v1.1.php
} 


\section{Preliminaries}

We formally define an entity collection as a collection of extracted text spans tied to named entity mentions. We denote these named entity mentions as $M=\left\{n e_{i}\right\}_{i=1}^{n}$, where $n e_{i}$ is the $i_{t h}$ named entity in the mention collection $M$ and $n$ is the size of $M$.

Cross-lingual entity lexicon creation seeks to create two entity collections $M_{1}$ and $M_{2}$ in a source and target language respectively. These two collections should be generated such that for each entity mention in $n e_{i} \in M_{1}$ in the source language, there is a corresponding named entity $n e_{j} \in M_{2}$ in the target language such that $n e_{i}$ and $n e_{j}$ refer to the same named entity in their respective language.

\section{Mining Cross-lingual Entities}

We introduce our approach to automatically extract cross-lingual entity pairs from large mined corpora.

\subsection{High-Resource NER}

We begin with large collections of comparable bitexts mined from large multilingual web corpora (ElKishky et al., 2020b). In particular, we select three mined web corpora 1) CCAligned (El-Kishky et al., 2020a), 2) WikiMatrix (Schwenk et al., 2019a), and 3) CCMatrix (Schwenk et al., 2019b)) due to the wide diversity of language pairs available in these mined corpora. We select language pairs of the form English-Target and tag each English sentence with named entity tags (Ramshaw and Marcus, 1999) using a pretrained NER tagger provided in the Stanza NLP toolkit ${ }^{3}$ (Qi et al., 2020). This NER model adopts a contextualized string representationbased tagger proposed by Akbik et al. (2018) and utilizes a forward and backward character-level LSTM language model. At tagging time, the representation at the end of each word position from both language models with word embeddings is fed into a standard Bi-LSTM sequence tagger with a conditional-random-field decoder.

\subsection{Entity Projection via Word Alignment}

We introduce three approaches for projecting entities and LSP-Align which combines all three.

\subsubsection{Lexical Alignment}

To perform word alignment using lexicalcooccurences, we apply FastAlign (Dyer et al., 2013), a fast loglinear re-parameterization of IBM

\footnotetext{
${ }^{3}$ https: //stanfordnlp.github.io/stanza/
}

Model 2 (Brown et al., 1993) and symmetrize alignments using the grow-diagonal-final-and (GDFA) heuristic.

FastAlign performs unsupervised word alignment over the full collection of mined bitexts using an expectation maximization based algorithm. While FastAlign is state-of-the-art in word alignment, due to its reliance on lexical co-occurences, it may misalign low-frequency entities.

\subsubsection{Semantic Alignment}

We leverage multilingual representations (embeddings) from the LASER toolkit (Artetxe and Schwenk, 2019) to align words that are semantically close. We propose a simple greedy word alignment algorithm guided by a distance function between words:

$$
\operatorname{sem}\left(w_{s}, w_{t}\right)=1-\frac{\mathbf{v}_{s} \cdot \mathbf{v}_{t}}{\left\|\mathbf{v}_{s}\right\|\left\|\mathbf{v}_{t}\right\|}
$$

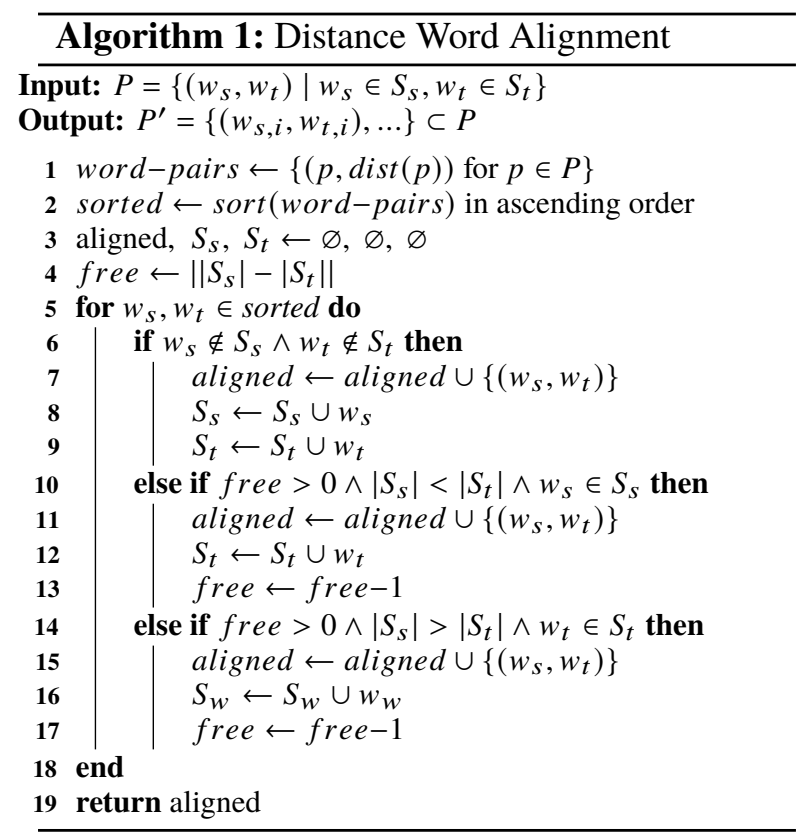

where Equation 1 shows that the semantic distances between a source word $\left(w_{s}\right)$ and target word $\left(w_{t}\right)$ is simply 1 minus the cosine similarity between $v_{s}$ and $v_{t}$, the LASER vector representations of $w_{s}$ and $w_{t}$ respectively. As shown in Algorithm 1, we take each source-target sentence pair and perform alignment between their tokens guided by the semantic distances between words. Of course, as source and target sentences, may be of different sizes, tokens in the shorter sentence may be aligned with multiple target tokens. Unlike lexical alignment with FastAlign, our distance-based alignment is deterministic and only needs a single pass through the bitexts. 


\subsubsection{Phonetic Alignment}

Recognizing that in many cases, phonetic transliterations are the avenue by which proper names travel between languages, we propose using phonetic signals to perform alignment and match named entities.

To align words based on their phonetic similarity, we leverage the distances between their transliterations and align words between the source and target that are "close" in this phonetic space. We adopt an unsupervised transliteration system developed by (Chen and Skiena, 2016) to transliterate between source and target languages and utilize Levenshtein distance (aka edit distance) (Wagner and Fischer, 1974) to calculate distances between transliterated words:

$$
\operatorname{phon}\left(w_{s}, w_{t}\right)=\min \left\{\begin{array}{l}
\left.\operatorname{LD}\left(T_{w_{s}}, w_{t}\right)\right) / \max \left(\left|T_{w_{s}}\right|,\left|w_{t}\right|\right) \\
\left.\operatorname{LD}\left(w_{s}, T_{w_{t}}\right)\right) / \max \left(\left|w_{s}\right|,\left|T_{w_{t}}\right|\right) \\
\operatorname{LD}\left(w_{s}, w_{t}\right) / \max \left(\left|w_{s}\right|,\left|w_{t}\right|\right)
\end{array}\right\}
$$

where $L D(\cdot, \cdot)$ is the Levenshtein distance between two strings and $T_{a}$ is the transliteration of word $a$ into word $b$ 's language. Equation 2 selects the minimum normalized distance between a source transliteration, target transliteration, and no transliteration to guide Algorithm 1 for a greedy word alignment. Once again, only a single pass over the data is required for alignment.

\subsubsection{Estimating Translation Probabilities}

Leveraging lexical alignment (i.e, FastAlign) alongside semantic and phoentic alignment yields three potential word alignments for a bitext collection. For alignment method $k$, we can iterate through the alignments and compute the counts of sourceto-target $(s, t)$ word pairings; we denote this count $\operatorname{cnt}(s, t)$. We can estimate the maximum likelihood translation probability from $s$ to $t$ given by alignment method $k$ as follows:

$$
\theta_{k, s, t}=\frac{\operatorname{cnt}(s, t)}{\sum_{t^{\prime}} \operatorname{cnt}\left(s, t^{\prime}\right)}
$$

Using Equation 3, we can compute the translation probabilities for lexical, semantic, and phonetic alignments which we use in our LSP-Align model.

\subsection{LSP Named-entity Projection}

We describe LSP-Align, which combines the three alignment signals for better entity-pair mining.
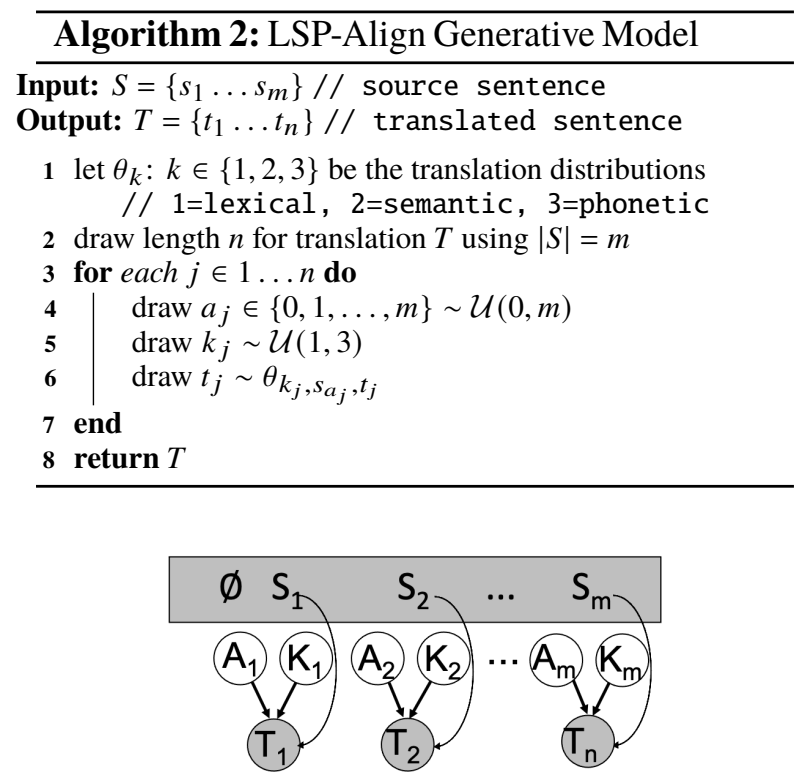

Figure 2: $\mathrm{S}$ and $\mathrm{T}$ are source/target sentences; target words are drawn from a distribution determined by (1) alignment, (2) source word, and (3) translation method

As described in Algorithm 2, the generative process takes in a source sentence $S$ and translates this sentence into the target sentence by drawing an alignment variable and translation mechanism (lexical, semantic, or phonetic) for each position in the target sentence and drawing a translated word from the corresponding translation distribution.

The graphical model for LSP-Align depicted in Figure 2, is similar to IBM-1 (Brown et al., 1993). The main difference is that, in addition to latent alignment variables $A$, we introduce latent translation mechanisms $K$. The translation distribution $\theta_{K, s}$ is chosen based on the latent alignment and mechanism variables. As we demonstrate in Equation 3, we can leverage the alignments for each alignment signal to estimate $\theta_{K, s}$ for each translation distribution. Using these estimated distributions in our model, we can infer the alignment variables as follows:

$$
\begin{aligned}
P\left(a_{j}=i \mid S, T, \theta\right) & =\sum_{k_{j}=1}^{3} P\left(a_{j}=i \mid S, T, k_{j}, \theta\right) \cdot P\left(k_{j}\right) \\
& =\sum_{k_{j}=1}^{3} \theta_{k_{j}, s_{i}, t_{j}} \cdot \frac{1}{3}
\end{aligned}
$$

where we assign the most probable alignment variable to each target word after marginalizing over the latent translation mechanisms (lexical, semantic, phonetic), which, for simplicity, we give equal probability. 


\begin{tabular}{llllcccc}
\hline Resource & Language & Num Bitexts & Distinct Ents & Lexical & Semantic & Phonetic & LSP-Align \\
\hline \multirow{2}{*}{ High } & Russian & $3.2 \mathrm{M}$ & $40.4 \mathrm{~K}$ & 0.84 & 0.81 & 0.83 & $\mathbf{0 . 8 6}$ \\
& Chinese & $5.2 \mathrm{M}$ & $28.4 \mathrm{~K}$ & $\mathbf{0 . 8 5}$ & 0.78 & 0.73 & $\mathbf{0 . 8 5}$ \\
& Turkish & $2.5 \mathrm{M}$ & $27.4 \mathrm{~K}$ & 0.88 & 0.89 & 0.87 & $\mathbf{0 . 9 0}$ \\
\hline \multirow{2}{*}{ Mid } & Arabic & $4.9 \mathrm{M}$ & $26.4 \mathrm{~K}$ & $\mathbf{0 . 8 8}$ & 0.80 & 0.81 & $\mathbf{0 . 8 8}$ \\
& Hindi & $1.2 \mathrm{M}$ & $7.60 \mathrm{~K}$ & 0.89 & 0.73 & 0.87 & $\mathbf{0 . 9 0}$ \\
& Romanian & $2.1 \mathrm{M}$ & $26.2 \mathrm{~K}$ & 0.93 & $\mathbf{0 . 9 4}$ & 0.92 & $\mathbf{0 . 9 4}$ \\
\hline \multirow{2}{*}{ Low } & Estonian & $1.3 \mathrm{M}$ & $15.2 \mathrm{~K}$ & 0.87 & $\mathbf{0 . 8 9}$ & 0.87 & $\mathbf{0 . 8 9}$ \\
& Armenian & $52 \mathrm{~K}$ & $2.30 \mathrm{~K}$ & 0.78 & 0.44 & $\mathbf{0 . 8 3}$ & 0.81 \\
& Tamil & $45 \mathrm{~K}$ & $2.50 \mathrm{~K}$ & 0.67 & 0.50 & 0.71 & $\mathbf{0 . 7 2}$ \\
\hline
\end{tabular}

Table 1: Fuzzy-F1 scores of mined cross-lingual entity pairs evaluated against gold-standard pairs.

\section{Experiments \& Results}

Datasets We utilize a gold standard evaluation lexicon created by (Pan et al., 2017) that leverages eight named parallel entity corpora ${ }^{4}$. We select nine languages from a diverse set of resource availability, language families, and scripts for evaluation.

Evaluation Protocol We evaluated the performance of the methods using the commonly used fuzzy-f1 score (Tsai and Roth, 2018) which is defined as the harmonic mean of the fuzzy precision and fuzzy recall scores. This metric is based on the longest common subsequence between a gold and mined entity, and has been used for several years in the NEWS transliteration workshops (Li et al., 2009; Banchs et al., 2015). The fuzzy precision and recall between a predicted string $p$ and the correct string $t$ is computed as follows:

$$
\begin{aligned}
\operatorname{fuzzy}-\operatorname{precision}(p, t) & =|\operatorname{LCS}(p, t)| /|p|, \\
\operatorname{fuzzy}-\operatorname{recall}(p, t) & =|\operatorname{LCS}(p, t)| /|t|,
\end{aligned}
$$

where $\operatorname{LCS}(\cdot, \cdot)$ is the longest common subsequence between two strings.

\subsection{Cross-lingual Entity Extraction}

We take a small sample of parallel sentences for each language, mine entity pairs using each projection technique, and compute Fuzzy-F1 using the gold-standard as a reference. As seen in Table 1, while lexical alignment outperforms semantic alignment, it displays similar performance to phonetic with phonetic performing better on low-resource languages and lexical performing better on highresource. However, LSP-Align outperforms or matches lexical alignment consistently showing that using all signals yields superior NE projection.

${ }^{4}$ Chinese-English Wikinames, Geonames, JRC names, LORELEI LRLP, NEWS 2015 task, Wikipedia names, Wikipedia places, and Wikipedia titles

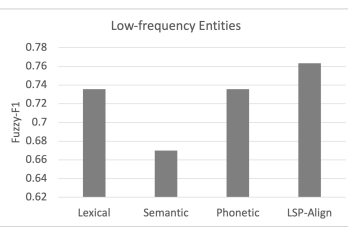

(a) low-frequency

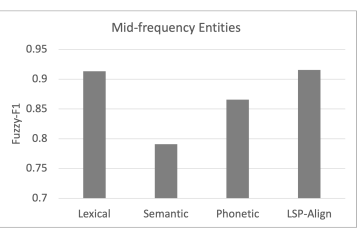

(b) mid-frequency

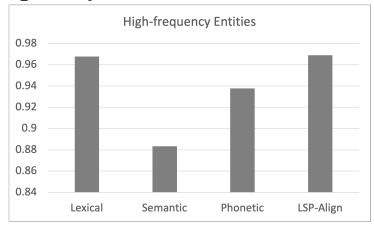

(c) high-frequency

Figure 3: Fuzzy-F1 by entity-frequency

Figure 3, separates the evaluated entities by frequency in the web-data bitexts (low $=0-3$, $\mathrm{mid}=4-10$, high $=11+$ ), and shows LSP-Align outperforming FastAlign when the entity is infrequent in the corpus. However, as entity frequency follows a long-tailed distribution, most entity mentions are infrequent.

In Table 2, we evaluate the quality of our full XLEnt dataset. As a general trend, the quality of extracted entities is high-resource $>$ mid-resource $>$ low-resource. This is intuitive as there are more parallel sentences that are likely better aligned on a sentence-level yielding better word alignments.

In Figure 4, we show that filtering on a higher mined frequency improves the overall quality of the entity pairs (albeit yielding a smaller dictionary). This is also intuitive as the redundancy of an entity pair being mined multiple times in different sentence pairs signals it's likely a true translation. This suggests that tuning the frequency threshold can be a useful tool to control the quality of the resultant entity lexicon.

\section{Conclusion}

We propose a technique that combines lexical alignment, semantic alignment, and phonetic alignment 


\begin{tabular}{lllll}
\hline Lang & Mined & P & R & F1 \\
\hline es & $9.2 \mathrm{M}$ & 0.91 & 0.89 & 0.90 \\
fr & $8.2 \mathrm{M}$ & 0.89 & 0.87 & 0.88 \\
ru & $7.9 \mathrm{M}$ & 0.62 & 0.59 & 0.61 \\
ja & $6.4 \mathrm{M}$ & 0.57 & 0.58 & 0.57 \\
zh & $6.3 \mathrm{M}$ & 0.38 & 0.38 & 0.38 \\
nl & $6.3 \mathrm{M}$ & 0.89 & 0.88 & 0.89 \\
it & $6.1 \mathrm{M}$ & 0.90 & 0.88 & 0.89 \\
ar & $5.8 \mathrm{M}$ & 0.78 & 0.77 & 0.77 \\
pt & $5.8 \mathrm{M}$ & 0.90 & 0.89 & 0.90 \\
pl & $5.5 \mathrm{M}$ & 0.85 & 0.85 & 0.85 \\
id & $4.2 \mathrm{M}$ & 0.90 & 0.89 & 0.90 \\
de & $4.1 \mathrm{M}$ & 0.87 & 0.85 & 0.86 \\
cs & $3.9 \mathrm{M}$ & 0.86 & 0.88 & 0.87 \\
tr & $3.8 \mathrm{M}$ & 0.87 & 0.84 & 0.86 \\
sv & $3.7 \mathrm{M}$ & 0.91 & 0.91 & 0.91 \\
uk & $3.7 \mathrm{M}$ & 0.75 & 0.68 & 0.72 \\
hu & $3.6 \mathrm{M}$ & 0.87 & 0.82 & 0.85 \\
ro & $3.3 \mathrm{M}$ & 0.90 & 0.89 & 0.90 \\
he & $3.2 \mathrm{M}$ & 0.81 & 0.75 & 0.78 \\
da & $3.0 \mathrm{M}$ & 0.92 & 0.92 & 0.92 \\
ca & $3.0 \mathrm{M}$ & 0.90 & 0.88 & 0.89 \\
el & $2.9 \mathrm{M}$ & 0.63 & 0.62 & 0.62 \\
ko & $2.9 \mathrm{M}$ & 0.38 & 0.40 & 0.39 \\
hr & $2.8 \mathrm{M}$ & 0.82 & 0.86 & 0.84 \\
fi & $2.6 \mathrm{M}$ & 0.83 & 0.89 & 0.86 \\
sk & $2.6 \mathrm{M}$ & 0.84 & 0.85 & 0.84 \\
bg & $2.5 \mathrm{M}$ & 0.79 & 0.80 & 0.79 \\
eo & $2.5 \mathrm{M}$ & 0.90 & 0.85 & 0.87 \\
no & $2.2 \mathrm{M}$ & 0.89 & 0.90 & 0.89 \\
hi & $2.0 \mathrm{M}$ & 0.73 & 0.74 & 0.73 \\
fa & $1.9 \mathrm{M}$ & 0.59 & 0.60 & 0.59 \\
ms & $1.8 \mathrm{M}$ & 0.85 & 0.79 & 0.82 \\
mk & $1.8 \mathrm{M}$ & 0.83 & 0.86 & 0.84 \\
et & $1.8 \mathrm{M}$ & 0.82 & 0.87 & 0.84 \\
gl & $1.7 \mathrm{M}$ & 0.88 & 0.89 & 0.89 \\
lt & $1.6 \mathrm{M}$ & 0.81 & 0.77 & 0.79 \\
bn & $1.6 \mathrm{M}$ & 0.61 & 0.66 & 0.64 \\
sr & $1.5 \mathrm{M}$ & 0.63 & 0.60 & 0.61 \\
\hline AVG & $3.8 \mathrm{M}$ & 0.79 & 0.79 & 0.79 \\
& & & \\
& & &
\end{tabular}

(a) High-resource languages.

\begin{tabular}{lllll}
\hline Lang & Mined & P & R & F1 \\
\hline sq & $1.4 \mathrm{M}$ & 0.90 & 0.86 & 0.88 \\
lv & $1.3 \mathrm{M}$ & 0.46 & 0.49 & 0.48 \\
th & $1.2 \mathrm{M}$ & 0.20 & 0.31 & 0.24 \\
tl & $1.1 \mathrm{M}$ & 0.81 & 0.78 & 0.79 \\
is & $960 \mathrm{~K}$ & 0.86 & 0.82 & 0.84 \\
xh & $960 \mathrm{~K}$ & 0.34 & 0.23 & 0.28 \\
sw & $870 \mathrm{~K}$ & 0.79 & 0.84 & 0.82 \\
sl & $860 \mathrm{~K}$ & 0.84 & 0.84 & 0.84 \\
eu & $800 \mathrm{~K}$ & 0.80 & 0.76 & 0.78 \\
ur & $746 \mathrm{~K}$ & 0.51 & 0.53 & 0.52 \\
ml & $739 \mathrm{~K}$ & 0.37 & 0.45 & 0.41 \\
si & $690 \mathrm{~K}$ & 0.66 & 0.67 & 0.67 \\
ast & $662 \mathrm{~K}$ & 0.58 & 0.62 & 0.60 \\
ta & $644 \mathrm{~K}$ & 0.30 & 0.30 & 0.30 \\
be & $582 \mathrm{~K}$ & 0.66 & 0.52 & 0.58 \\
mr & $548 \mathrm{~K}$ & 0.46 & 0.53 & 0.49 \\
ha & $437 \mathrm{~K}$ & 0.09 & 0.08 & 0.09 \\
mg & $320 \mathrm{~K}$ & 0.72 & 0.79 & 0.76 \\
ne & $319 \mathrm{~K}$ & 0.42 & 0.45 & 0.43 \\
lb & $312 \mathrm{~K}$ & 0.46 & 0.49 & 0.47 \\
az & $298 \mathrm{~K}$ & 0.67 & 0.68 & 0.67 \\
bs & $267 \mathrm{~K}$ & 0.83 & 0.84 & 0.83 \\
ka & $266 \mathrm{~K}$ & 0.48 & 0.47 & 0.47 \\
fy & $266 \mathrm{~K}$ & 0.65 & 0.70 & 0.67 \\
jv & $235 \mathrm{~K}$ & 0.59 & 0.64 & 0.62 \\
oc & $234 \mathrm{~K}$ & 0.68 & 0.74 & 0.71 \\
af & $234 \mathrm{~K}$ & 0.86 & 0.87 & 0.86 \\
cy & $223 \mathrm{~K}$ & 0.84 & 0.81 & 0.82 \\
hy & $216 \mathrm{~K}$ & 0.56 & 0.58 & 0.57 \\
ceb & $208 \mathrm{~K}$ & 0.32 & 0.39 & 0.35 \\
la & $198 \mathrm{~K}$ & 0.57 & 0.59 & 0.58 \\
ga & $171 \mathrm{~K}$ & 0.35 & 0.38 & 0.36 \\
kk & $162 \mathrm{~K}$ & 0.60 & 0.59 & 0.59 \\
sd & $148 \mathrm{~K}$ & 0.39 & 0.46 & 0.42 \\
te & $147 \mathrm{~K}$ & 0.66 & 0.67 & 0.67 \\
su & $144 \mathrm{~K}$ & 0.71 & 0.73 & 0.72 \\
yi & $118 \mathrm{~K}$ & 0.11 & 0.18 & 0.14 \\
ht & $115 \mathrm{~K}$ & 0.81 & 0.84 & 0.82 \\
\hline AVG & $502 \mathrm{~K}$ & 0.58 & 0.59 & 0.58 \\
& & &
\end{tabular}

(b) Mid-resource languages.

\begin{tabular}{lllll}
\hline Lang & Mined & $\mathbf{P}$ & $\mathbf{R}$ & $\mathbf{F 1}$ \\
\hline br & $109 \mathrm{~K}$ & 0.45 & 0.53 & 0.49 \\
mn & $92 \mathrm{~K}$ & 0.63 & 0.62 & 0.62 \\
km & $79 \mathrm{~K}$ & 0.37 & 0.46 & 0.41 \\
ilo & $79 \mathrm{~K}$ & 0.55 & 0.63 & 0.59 \\
am & $72 \mathrm{~K}$ & 0.63 & 0.66 & 0.65 \\
so & $70 \mathrm{~K}$ & 0.64 & 0.64 & 0.64 \\
ig & $70 \mathrm{~K}$ & 0.67 & 0.69 & 0.68 \\
my & $60 \mathrm{~K}$ & 0.43 & 0.35 & 0.38 \\
gd & $58 \mathrm{~K}$ & 0.24 & 0.23 & 0.23 \\
nds & $57 \mathrm{~K}$ & 0.67 & 0.67 & 0.67 \\
ps & $55 \mathrm{~K}$ & 0.64 & 0.60 & 0.62 \\
yo & $51 \mathrm{~K}$ & 0.75 & 0.70 & 0.73 \\
fo & $35 \mathrm{~K}$ & 0.76 & 0.79 & 0.77 \\
tt & $33 \mathrm{~K}$ & 0.36 & 0.40 & 0.38 \\
ba & $31 \mathrm{~K}$ & 0.44 & 0.43 & 0.44 \\
kn & $31 \mathrm{~K}$ & 0.39 & 0.41 & 0.40 \\
gu & $31 \mathrm{~K}$ & 0.52 & 0.52 & 0.52 \\
pa & $29 \mathrm{~K}$ & 0.45 & 0.44 & 0.45 \\
lo & $29 \mathrm{~K}$ & 0.40 & 0.37 & 0.38 \\
an & $29 \mathrm{~K}$ & 0.74 & 0.76 & 0.75 \\
zu & $28 \mathrm{~K}$ & 0.72 & 0.64 & 0.68 \\
bar & $25 \mathrm{~K}$ & 0.64 & 0.67 & 0.66 \\
or & $23 \mathrm{~K}$ & 0.13 & 0.17 & 0.15 \\
arz & $22 \mathrm{~K}$ & 0.56 & 0.60 & 0.58 \\
wuu & $20 \mathrm{~K}$ & 0.06 & 0.32 & 0.11 \\
lmo & $20 \mathrm{~K}$ & 0.77 & 0.78 & 0.77 \\
io & $18 \mathrm{~K}$ & 0.85 & 0.88 & 0.86 \\
tg & $16 \mathrm{~K}$ & 0.34 & 0.36 & 0.35 \\
mwl & $14 \mathrm{~K}$ & 0.76 & 0.75 & 0.75 \\
wo & $8 \mathrm{~K}$ & 0.29 & 0.28 & 0.29 \\
ff & $7 \mathrm{~K}$ & 0.26 & 0.14 & 0.18 \\
ug & $5 \mathrm{~K}$ & 0.15 & 0.18 & 0.16 \\
tn & $5 \mathrm{~K}$ & 0.69 & 0.66 & 0.67 \\
as & $3 \mathrm{~K}$ & 0.37 & 0.39 & 0.38 \\
ln & $3 \mathrm{~K}$ & 0.27 & 0.28 & 0.27 \\
ss & $2 \mathrm{~K}$ & 0.55 & 0.44 & 0.48 \\
om & $1 \mathrm{~K}$ & 0.25 & 0.20 & 0.22 \\
lg & $1 \mathrm{~K}$ & 0.22 & 0.19 & 0.20 \\
\hline AVG & $35 \mathrm{~K}$ & 0.49 & 0.50 & 0.49 \\
& & & &
\end{tabular}

(c) Low-resource languages.

Table 2: Fuzzy metrics of extracted cross-lingual pairs evaluated against gold entity pairs.

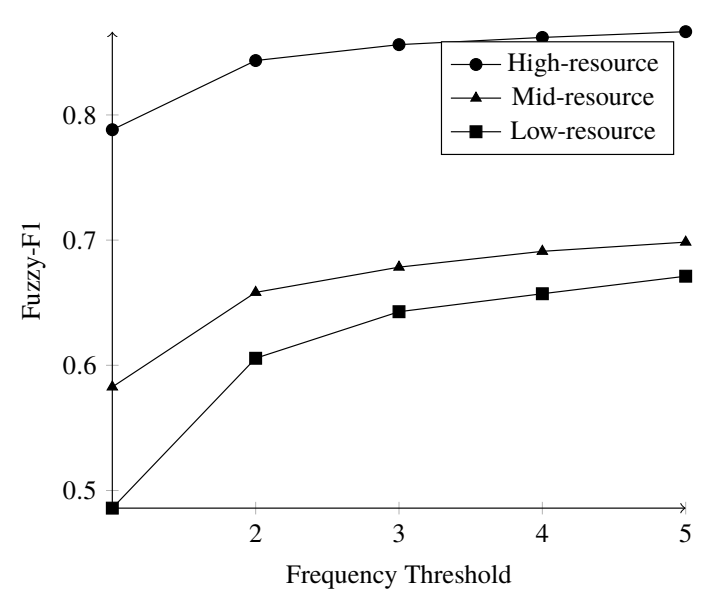

Figure 4: Fuzzy-F1 for high, mid, and low-resource languages for different mined frequency thresholds. into a unified alignment model. We demonstrate this unified model better extracts cross-lingual entity pairs than any single alignment. Leveraging this model, we automatically curate a large, crosslingual entity lexicon covering 120 languages paired with English which we freely release to the community. Accompanying this lexicon, we release a large multilingual collection of sentences tagged via named-entity projection. We hope these resources facilitate future multilingual NLP work such as multilingual NER, multilingual entity linking, and multilingual knowledge base construction.

\section{References}

Alan Akbik, Duncan Blythe, and Roland Vollgraf. 2018. Contextual string embeddings for sequence labeling. 
In Proceedings of the 27th International Conference on Computational Linguistics, pages 1638-1649.

Mikel Artetxe and Holger Schwenk. 2019. Massively multilingual sentence embeddings for zeroshot cross-lingual transfer and beyond. Transactions of the Association for Computational Linguistics, 7:597-610

Rafael E Banchs, Min Zhang, Xiangyu Duan, Haizhou $\mathrm{Li}$, and A Kumaran. 2015. Report of news 2015 machine transliteration shared task. In Proceedings of the Fifth Named Entity Workshop, pages 10-23.

Peter F Brown, Stephen A Della Pietra, Vincent J Della Pietra, and Robert L Mercer. 1993. The mathematics of statistical machine translation: Parameter estimation. Computational linguistics, 19(2):263311 .

Yanqing Chen and Steven Skiena. 2016. False-friend detection and entity matching via unsupervised transliteration. arXiv preprint arXiv:1611.06722.

Ryan Cotterell and Kevin Duh. 2017. Lowresource named entity recognition with cross-lingual, character-level neural conditional random fields. In Proceedings of the Eighth International Joint Conference on Natural Language Processing (Volume 2: Short Papers), pages 91-96.

Dipanjan Das and Slav Petrov. 2011. Unsupervised partof-speech tagging with bilingual graph-based projections. In Proceedings of the 49th Annual Meeting of the Association for Computational Linguistics: $\mathrm{Hu}$ man Language Technologies, pages 600-609.

Chris Dyer, Victor Chahuneau, and Noah A Smith 2013. A simple, fast, and effective reparameterization of ibm model 2. In Proceedings of the 2013 Conference of the North American Chapter of the Association for Computational Linguistics: Human Language Technologies, pages 644-648.

Ahmed El-Kishky, Vishrav Chaudhary, Francisco Guzman, and Philipp Koehn. 2020a. Ccaligned: A massive collection of cross-lingual web-document pairs. In EMNLP.

Ahmed El-Kishky, Philipp Koehn, and Holger Schwenk 2020b. Searching the web for cross-lingual parallel data. In Proceedings of the 43rd International ACM SIGIR Conference on Research and Development in Information Retrieval, pages 2417-2420.

Xiaocheng Feng, Xiachong Feng, Bing Qin, Zhangyin Feng, and Ting Liu. 2018. Improving low resource named entity recognition using cross-lingual knowledge transfer. In IJCAI, pages 4071-4077.

Seokhwan Kim, Minwoo Jeong, Jonghoon Lee, and Gary Geunbae Lee. 2010. A cross-lingual annotation projection approach for relation detection. In Proceedings of the 23rd International Conference on Computational Linguistics (Coling 2010), pages 564-571.
Haizhou Li, A Kumaran, Vladimir Pervouchine, and Min Zhang. 2009. Report of news 2009 machine transliteration shared task. In Proceedings of the 2009 Named Entities Workshop: Shared Task on Transliteration (NEWS 2009), pages 1-18.

Jian Ni, Georgiana Dinu, and Radu Florian. 2017. Weakly supervised cross-lingual named entity recognition via effective annotation and representation projection. In Proceedings of the 55th Annual Meeting of the Association for Computational Linguistics (Volume 1: Long Papers), pages 1470-1480.

Xiaoman Pan, Boliang Zhang, Jonathan May, Joel Nothman, Kevin Knight, and Heng Ji. 2017. Crosslingual name tagging and linking for 282 languages. In Proceedings of the 55th Annual Meeting of the Association for Computational Linguistics (Volume 1: Long Papers), pages 1946-1958.

Peng Qi, Yuhao Zhang, Yuhui Zhang, Jason Bolton, and Christopher D. Manning. 2020. Stanza: A Python natural language processing toolkit for many human languages. In Proceedings of the 58th Annual Meeting of the Association for Computational Linguistics: System Demonstrations.

Lance A Ramshaw and Mitchell P Marcus. 1999. Text chunking using transformation-based learning. In Natural language processing using very large corpora, pages 157-176. Springer.

Xiang Ren, Ahmed El-Kishky, Heng Ji, and Jiawei Han 2016a. Automatic entity recognition and typing in massive text data. In Proceedings of the 2016 International Conference on Management of Data, pages 2235-2239.

Xiang Ren, Ahmed El-Kishky, Chi Wang, and Jiawei Han. 2016b. Automatic entity recognition and typing in massive text corpora. In Proceedings of the 25th International Conference Companion on World Wide Web, pages 1025-1028.

Holger Schwenk, Vishrav Chaudhary, Shuo Sun, Hongyu Gong, and Francisco Guzmán. 2019a. Wikimatrix: Mining $135 \mathrm{~m}$ parallel sentences in 1620 language pairs from wikipedia. arXiv preprint arXiv:1907.05791.

Holger Schwenk, Guillaume Wenzek, Sergey Edunov, Edouard Grave, and Armand Joulin. 2019b. Ccmatrix: Mining billions of high-quality parallel sentences on the web. arXiv preprint arXiv:1911.04944.

Satoshi Sekine and Elisabete Ranchhod. 2009. Named entities: recognition, classification and use, volume 19. John Benjamins Publishing.

Fangbo Tao, George Brova, Jiawei Han, Heng Ji, Chi Wang, Brandon Norick, Ahmed El-Kishky, Jialu Liu, Xiang Ren, and Yizhou Sun. 2014. Newsnetexplorer: Automatic construction and exploration of news information networks. In Proceedings of the 2014 ACM SIGMOD International Conference on Management of Data, pages 1091-1094. 
Chen-Tse Tsai and Dan Roth. 2018. Learning better name translation for cross-lingual wikification. In AAAI.

Robert A Wagner and Michael J Fischer. 1974. The string-to-string correction problem. Journal of the $A C M(J A C M), 21(1): 168-173$.

Mengqiu Wang, Wanxiang Che, and Christopher D Manning. 2013. Joint word alignment and bilingual named entity recognition using dual decomposition. In Proceedings of the 51st Annual Meeting of the Association for Computational Linguistics (Volume 1: Long Papers), pages 1073-1082.

Mengqiu Wang and Christopher D Manning. 2014. Cross-lingual projected expectation regularization for weakly supervised learning. Transactions of the Association for Computational Linguistics, 2:55-66.

Jiateng Xie, Zhilin Yang, Graham Neubig, Noah A Smith, and Jaime G Carbonell. 2018. Neural crosslingual named entity recognition with minimal resources. In Proceedings of the 2018 Conference on Empirical Methods in Natural Language Processing, pages 369-379. 